\title{
Enunciados de Tarefas de Matemática Baseados na Perspectiva da Educação Matemática Realística
}

\author{
Math Tasks Instructions Based on the Realistic Mathematics \\ Education Perspective
}

\author{
Pamela Emanueli Alves Ferreira* \\ Regina Luzia Corio de Buriasco ${ }^{* *}$
}

\begin{abstract}
Resumo
Este artigo apresenta um estudo a respeito de enunciados de tarefas de Matemática na perspectiva da Educação Matemática Realística que permite: analisá-las no que diz respeito à sua classificação, às suas características, potencialidades e constituição; saber se a tarefa é rotineira ou não, a que tipo de situação e item remete, se oportuniza matematização, se a tarefa é flexível, se permite diferentes estratégias de resolução, que tipo de competências promove, se é caracterizada como exercício ou problema. Uma intenção subjacente é que este trabalho sirva como um recurso para professores que ensinam Matemática reconhecerem potencialidades e limitações em tarefas de Matemática para utilizá-las em um ambiente de avaliação como prática de investigação.
\end{abstract}

Palavras-chave: Educação Matemática. Educação Matemática Realística. Enunciados de Tarefas de Matemática. Contexto. Avaliação como Prática de Investigação.

\begin{abstract}
The objective of this article is to present a study on Math tasks instructions based on the Realistic Mathematics Education perspective, which allows: a) the analysis of Math tasks regarding their classification, characteristics, potentialities and constitution; b) to know whether the task is carried out routinely or not, what type of situation is refers to, whether it promotes mathematization processes, whether the task is flexible, whether it allows different resolution strategies, what kind of competencies it promotes, and whether it is characterized as an exercise or problem. An underlying intention of this research is to work as a resource for Math teachers, helping them understand Math tasks, analyze their potentials and limitations, and use them in the context of assessment as an investigative practice.
\end{abstract}

Key words: Mathematics Education. Realistic Mathematics Education. Mathematics Tasks Instructions. Context. Assessment as an Investigative Practice.

\footnotetext{
* Doutora em Ensino de Ciências e Educação Matemática pela Universidade Estadual de Londrina (UEL). Docente do Depto. de Matemática da Universidade Estadual de Londrina (UEL), Londrina-PR, Brasil. Endereço para correspondencia: Rua Prof. Couto da Costa, 303, Vila Mesquita, Cambé, PR, Brasil, CEP: 86182-490. Email: pamelauel@gmail.com. Este artigo é parte da tese de doutorado da primeira autora, bolsista da CAPES.

** Doutora em Educação pela Universidade Estadual Paulista "Júlio de Mesquita Filho" (UNESP). Docente do Depto. de Matemática e do Programa de Pós-Graduação em Ensino de Ciências e Educação Matemática da Universidade Estadual de Londrina (UEL), Londrina-PR, Brasil. Endereço para correspondencia: R. Eduardo Benjamin Hosken, 173, apto. 501, Jardim Conceição, Londrina-PR, Brasil, CEP: 86020-440. E-mail: reginaburiasco@gmail.com. Bolsista do CNPq.
} 


\section{Considerações preliminares}

Na reflexão a respeito das informações que os alunos apresentam em situações de aula ou de prova, mais especificamente na busca da compreensão das formas como os alunos lidam com tarefas de Matemática, deve-se levar em consideração o papel relevante que os enunciados das tarefas propostas exercem.

Estudos desenvolvidos pelo GEPEMA ${ }^{1}$ que tiveram como objeto a "avaliação como prática de investigação" (BURIASCO; FERREIRA; CIANI, 2009; FERREIRA, 2009) e a análise da produção escrita e, como meta, conhecer as formas pelas quais alunos e professores lidavam com as tarefas propostas revelam que uma das principais dificuldades apresentadas pelos estudantes refere-se à interpretação de seus enunciados em situação de avaliação. Por considerar relevante conhecer o papel que os contextos podem desempenhar nas tarefas dos estudantes, apresentamos um estudo a respeito de enunciados de tarefas matemáticas na busca para conhecer suas classificações, características, potencialidades, constituição.

Considerando que, por um lado, o contexto envolvido em uma tarefa exerce um papel importante (TREFFERS; GOFFREE, 1985; DE LANGE, 1987; TREFFERS, 1987; VAN DEN HEUVEL-PANHUIZEN, 1996, 2005; SHANNON, 2007), sendo, por vezes, um aspecto que pode determinar o sucesso, ou não, dos estudantes em suas resoluções (CLEMENTS, 1980; VAN DEN HEUVEL-PANHUIZEN, 1996, 2005) e, por outro, que as tarefas de sala de aula não devem ser diferentes das de avaliação, e que esta deveria mostrar indícios da aprendizagem do estudante, fez-se relevante um estudo do que as tarefas podem oportunizar, isso na perspectiva de uma Educação Matemática que leva em conta a fenomenologia didática $^{2}$, a matematização, a reinvenção guiada ${ }^{3}$.

Nessa direção, a pesquisa (FERREIRA, 2013) que gerou este artigo buscou apresentar um quadro de referência para a leitura de enunciados de tarefas matemáticas e, no sentido de conhecer suas características, seguiu uma abordagem qualitativa de cunho interpretativo sob as orientações presentes na análise de conteúdo (BARDIN, 2004), à luz de pressupostos teóricos da Educação Matemática Realística como quadro de referência para a análise dos

\footnotetext{
${ }^{1}$ GEPEMA - Grupo de Estudos e Pesquisa em Educação Matemática e Avaliação, da Universidade Estadual de Londrina (UEL). Outras informações em: http://www.uel.br/grupo-estudo/gepema/index.html.

2 A fenomenologia didática, segundo Freudenthal (1983), se mostra como uma maneira de o professor oportunizar aos alunos os "lugares" ou "situações" pelas quais podem reinventar "suas" matemáticas, matematizar.

${ }^{3}$ Neste artigo tomamos como base pressuspostos teóricos da Educação Matemática Realística - uma abordagem de ensino e aprendizagem cujo desenvolvimento foi inspirado, principalmente, pelas ideias e contribuições do educador matemático alemão Hans Freudenthal (1905-1990) (FERREIRA, 2013).
} 
enunciados e contextos de tarefas de Matemática presentes em um livro didático.

\section{Os contextos das tarefas e suas classificações}

Partindo do pressuposto de que a avaliação escolar deve fornecer informações a respeito da aprendizagem dos estudantes, devemos pensar em instrumentos de avaliação que contenham tarefas que possibilitem aos estudantes apresentar essas informações. Para tanto, a flexibilidade $^{4}$ da tarefa, a pergunta, o contexto, a forma de apresentação são assuntos que merecem ser estudados.

A ênfase que a RME (Realistic Mathematics Education - Educação Matemática Realística) coloca no contexto das tarefas matemáticas, que envolvem situações por meio das quais os estudantes possam imaginá-las, torná-las reais em suas mentes, realizá-las, é o que dá o nome à abordagem realística da educação matemática preconizada por Hans Freudenthal (FREUDENTHAL, 1983). Os contextos, nessa perspectiva, parecem ser a matéria-prima da matematização.

O contexto de uma tarefa pode apresentar situações realísticas, fantasiosas, factuais, ou pode até mesmo ser estritamente circunscrito por uma linguagem matemática (VAN DEN HEUVEL-PANHUIZEN, 1996). O contexto pode ser um potencializador para a oportunidade de matematizar. O fato de um contexto integrar uma situação do cotidiano não é suficiente para que o estudante possa aprender algo ao lidar com ele. Com isso, não é possível dizer a priori quais seriam bons problemas de contexto, visto que essa caracterização depende da relação que o resolvedor em potencial estabelece com o enunciado. Todavia, a hipótese é de que a proximidade do contexto com o repertório do estudante aumenta a possibilidade de matematização ${ }^{5}$.

Para Borasi (1986), um dos principais papéis do contexto para a realização de uma tarefa é o de fornecer ao resolvedor as informações que possam permitir sua resolução. Também argumenta que experiências na resolução de problemas da vida real poderiam

\footnotetext{
${ }^{4}$ Uma tarefa é considerada flexível quando permite resolução em diferentes níveis de complexidade e/ou diferentes estratégias. Por outro lado, uma tarefa inflexível é aquela que, geralmente, dá margem para uma única resolução, muitas vezes padronizada.

5 “[...] uma atividade organizada. Ela refere-se à essência da atividade matemática, à linha que atravessa toda educação matemática voltada para a elaboração de conhecimento factual, à aprendizagem de conceitos, à obtenção de habilidades e ao uso da linguagem e de outras organizações, às habilidades na resolução de problemas que estão, ou não, em um contexto matemático" (TREFFERS, 1987, p. 51-52, tradução nossa).
} 
contribuir para o desenvolvimento de diferentes estratégias, para analisar contextos, para elaborar formulações matemáticas.

Clements (1980) chamou a atenção para a necessidade de pesquisas a respeito dos fatores que fazem com que alguns problemas aritméticos verbais sejam mais acessíveis do que os correspondentes aritméticos estruturados, apesar de envolverem mais leitura, compreensão e transformação. Sua pesquisa revelou que, em um teste com 126 estudantes, 58 acertaram o item $^{6}$ a seguir "Questão 5 - Escrevam a resposta correta para $1-1 / 4$ ”, enquanto 98 acertaram o problema aritmético verbal correspondente "Questão 18 - Um bolo é cortado em quatro partes iguais e Bill leva uma das partes. Que fração do bolo resta?" (CLEMENTS, 1980, p. 19, tradução nossa).

Os problemas que apresentam situações contextuais não devem ser apresentados apenas em fase de aplicação, como é feito tradicionalmente, mas também em fase de desenvolvimento e exploração, pois fazem com que os estudantes reconheçam a utilidade da Matemática em suas necessidades e vida diária, além de despertarem a curiosidade e a criatividade (DÍAZ; POBLETE, 2005; VAN DEN HEUVEL-PANHUIZEN, 2001). Para Van den Heuvel-Panhuizen (2001), Problemas de Contexto e situações da vida real servem para constituir e aplicar conceitos matemáticos: enquanto trabalham com problemas de contexto, os alunos podem desenvolver ferramentas e compreensão matemática. Os Problemas de Contexto são definidos na RME como situações-problema que são experimentalmente reais para os estudantes (GRAVEMEIJER; DOORMAN, 1999).

Um problema de contexto, em geral, necessita de matematização, o que pode demandar alguma exploração do estudante que pretende resolvê-lo. Por outro lado, se for possível matematizar o problema de maneira quase automática e sem muitos esforços, não se trata de um problema de contexto, mas de um exercício de matematização (DÍAZ; POBLETE, 2005). Contextualizar o conhecimento matemático não significa simplesmente simulá-lo em sala de aula com qualquer atividade cotidiana, porém exige conhecer as representações que os alunos fazem desse conhecimento e conhecer o significado de suas concepções (DÍAZ; POBLETE, 2005). Díaz e Poblete (2005) apresentam uma classificação para tarefas ( problemas $^{7}$ ) segundo seu contexto:

Problema de contexto real - Um contexto é real se ele é produzido efetivamente na realidade e envolve ações do aluno.

\footnotetext{
${ }^{6}$ Usualmente, é chamado de item, ou questão, uma tarefa em circunstância de prova/teste.

7 Os autores fazem a classificação de "Problemas", mas achamos adequado chamá-los de tarefas.
} 
Problema de contexto realista ${ }^{8}$ - Um contexto é realista se ele pode realmente ocorrer. Trata-se de uma simulação de realidade ou de uma parte dela.

Problema de contexto fantasioso - Um contexto é fantasioso se for fruto da imaginação sem fundamento na realidade.

Problema de contexto puramente matemático - Um contexto é puramente matemático se se refere exclusivamente a objetos matemáticos: números, relações e operações aritméticas, figuras geométricas etc. (DÍAZ; POBLETE, 2005, p. 4, tradução nossa).

O papel dos contextos nas tarefas de avaliação é um assunto complexo que vai muito além de simplesmente motivar os estudantes a lidar com uma tarefa (SHANNON, 2007). Para a autora, a importância atribuída aos contextos das tarefas está associada mais à oportunidade que representam para a abstração matemática por meio de situações diversas e diferentes representações do que tornar o contexto matemático familiar aos estudantes. No entanto, ela reconhece que esse é um papel importante dos contextos para tornar o conhecimento matemático mais acessível a eles e ainda argumenta que o potencial de uma tarefa de contexto para gerar discussão e abstração depende do modo como ela é tratada. Além disso, por mais útil que a tarefa seja, pode ser inútil se os alunos não tiverem condições de lidar com a complexidade da Matemática intrínseca subjacente.

Problemas de contexto têm formas específicas, conteúdos e funções, podem ser editados em linguagem puramente aritmética, como problemas de palavra e texto, e serem apresentados por meios de jogos, histórias, noticiários, modelos, gráficos, ou ainda pela combinação de tais portadores de informações, agrupados em temas ou projetos (TREFFERS; GOFFREE, 1985). Dependendo da forma como o problema é utilizado, pode ser considerado como de contexto ou não (problema de palavra ${ }^{9}$ ).

Um dos princípios da RME é relativo ao papel que os contextos dos problemas desempenham na formação dos estudantes. De acordo com Treffers e Goffree (1985), esses contextos cumprem uma série de funções:

- formação de conceito: na fase inicial de um curso, permitirão aos alunos um acesso natural e motivador para a matemática;

- modelo de formação: eles fornecem um suporte seguro para a aprendizagem de operações formais, procedimentos, notações, regras, e fazem isso juntamente com outros modelos que têm uma função importante como suporte para o pensamento;

- aplicabilidade: eles descobrem a realidade como fonte e domínio de aplicações;

- exercício de habilidades específicas em situações aplicadas (TREFFERS; GOFFREE, 1985, p. 111; TREFFERS, 1987, p. 256, tradução e grifos nossos).

\footnotetext{
${ }^{8}$ Não é no mesmo sentido de realístico. Realista, nesse caso, está associado a contextos que dizem respeito a informações que são ou podem ser efetivamente reais, enquanto realístico diz respeito a contextos que são imagináveis, podendo ser da realidade ou fictícios.

${ }^{9}$ Problema de Palavra: são problemas que geralmente possuem todas as informações necessárias para resolver o problema, o contexto está explícito no texto do problema, envolve a combinação de algoritmos padrões e a solução é única e exata (BORASI, 1986).
} 
Os Problemas de Contexto são considerados como uma matéria-prima no que diz respeito ao Princípio da Realidade, o qual, dentre outros quatro/cinco princípios, caracteriza a Educação Matemática Realística, segundo educadores como Van den Heuvel-Panhuizen (1996, 2000, 2001, 2010), Treffers (1987) e Streefland (1991). Esse princípio concebe a realidade como uma fonte para a aprendizagem. Fundamentado na perspectiva da Matemática como atividade humana, assim como a Matemática tem origem na matematização da realidade, a natureza da aprendizagem matemática também tem sua origem na matematização da realidade ${ }^{10}$.

No ambiente pedagógico, matematizar a realidade significa explorar contextos ricos que demandam uma organização matemática ou, em outras palavras, contextos que podem ser matematizados. Se os estudantes vivenciarem o processo de reinventar a Matemática como uma expansão do que já conhecem, essa pode ser uma aproximação desejável entre suas experiências de vida cotidiana e a Matemática, pois ambas farão parte da mesma realidade (GRAVEMEIJER; DOORMAN, 1999).

Nesse sentido, espera-se que os estudantes, ao trabalhar com problemas de diferentes contextos, possam desenvolver ferramentas matemáticas, compreensão, estratégias que sejam intimamente ligadas ao contexto. Partindo da exploração de fenômenos diversos, por meio de estratégias menos informais, e progredindo no sentido de sistematizar e obter caráter de um modelo formal, por meio da matematização, certos aspectos do contexto matemático podem tornar-se mais gerais e fornecerem apoio para a resolução de outros problemas relacionados (VAN DEN HEUVEL-PANHUIZEN, 2001). A fim de cumprir a função de passagem entre um nível informal e o formal, os modelos que recebem caráter modelo de (específicos) passam a um modelo para (mais geral) (STREEFLAND, 1991).

No que diz respeito às possibilidades de matematização, De Lange (1987, p. 76-77) classifica diferentes usos/utilidades/fins/objetivos (uses) dos contextos (de terceira, segunda e primeira ordem), como segue.

\begin{tabular}{|c|c|}
\hline $\begin{array}{c}\text { Contexto de } \\
\text { Ordem } \\
\text { Zero }\end{array}$ & $\begin{array}{l}\text { É utilizado apenas para tornar o problema parecido com uma situação da vida } \\
\text { real. São chamados por De Lange (1999) de "contexto falso", "contexto de } \\
\text { camuflagem". Segundo o autor, os problemas que contêm esse tipo de } \\
\text { contexto devem ser evitados. Para Dekker e Querelle (2002), o contexto } \\
\text { utilizado em um problema deve ser relevante para resolvê-lo; caso contrário; } \\
\text { ele é classificado como de ordem zero. }\end{array}$ \\
\hline
\end{tabular}

\footnotetext{
${ }^{10}$ Nesse sentido, Freudenthal (1983) critica a inversão didática que geralmente é feita no ensino tradicional: em vez de partir do problema concreto e investigá-lo por meios matemáticos, a matemática vem em primeiro lugar, e o problema concreto vem depois como uma aplicação (FREUDENTHAL, 1983, p. 132).
} 


\begin{tabular}{|c|l|l|}
\hline $\begin{array}{c}\text { Contexto de } \\
\text { Primeira } \\
\text { Ordem }\end{array}$ & $\begin{array}{l}\text { É aquele que apresenta operações matemáticas "textualmente embaladas", no } \\
\text { qual uma simples tradução do enunciado para uma linguagem matemática é } \\
\text { suficiente (DE LANGE, 1987) Esse tipo de contexto é relevante e necessário } \\
\text { para resolver o problema e avaliar a resposta (DE LANGE, 1999; DEKKER; } \\
\text { QUERELLE, 2002). }\end{array}$ \\
\hline $\begin{array}{c}\text { Contexto de } \\
\text { Segunda } \\
\text { Ordem }\end{array}$ & $\begin{array}{l}\text { É aquele com o qual o estudante é confrontado com uma situação realística e } \\
\text { dele é esperado que encontre ferramentas matemáticas para organizar, } \\
\text { estruturar e resolver a tarefa (DE LANGE, 1987). Esse tipo de contexto, } \\
\text { segundo De Lange (1999), envolve matematização; ao passo que, nos } \\
\text { contextos de primeira ordem, os problemas já são pré-matematizados. }\end{array}$ \\
\hline $\begin{array}{c}\text { Contexto de } \\
\text { Terceira } \\
\text { Ordem }\end{array}$ & $\begin{array}{l}\text { Como aquele que possibilita um "processo de matematização conceitual", esse } \\
\text { tipo de contexto serve para "introduzir ou desenvolver um conceito ou modelo } \\
\text { matemático" (DE LANGE, 1987, p. 76). }\end{array}$ \\
\hline
\end{tabular}

Quadro 1 - Usos do contexto segundo De Lange (1995)

Fonte: Elaborado pelas autoras com base em De Lange (1995).

Uma das funções mais características da RME é o uso dos contextos para formação conceitual, tal aspecto está relacionado ao que se chama de "processo de matematização conceitual". De Lange (1995) explicita que, quando se trata de tarefas de prova escrita, essa função do contexto não é sempre utilizada, pelo fato de que, usualmente, não são introduzidos novos conceitos durante uma prova, mas são aplicados conceitos matemáticos de alguma forma. Por conseguinte, nas tarefas de avaliação são associadas, quase sempre, apenas as três primeiras classes de funcionalidades dos contextos.

A seguir são apresentados alguns exemplos de enunciados de tarefas, segundo a classe de funcionalidade do contexto, apresentados por De Lange (1995).

\begin{tabular}{|l|l|}
\hline Classe de funcionalidade de Contexto & \multicolumn{1}{c|}{ Exemplos } \\
\hline 1. Nenhuma função: não há contexto. & $\begin{array}{c}\sin ^{2} 30^{0}-\left(\frac{1}{2}\right)^{2} \cdot(0.8)^{-1}+\sqrt{2.25} \\
\frac{11}{22}+\left(\frac{2}{3}\right)^{2} \cdot\left(\cos 60^{0}+\tan 45^{0}\right)^{2}\end{array}$ \\
\hline $\begin{array}{l}\text { 2. O contexto é utilizado para camuflar } \\
\text { vestir o problema matemático. }\end{array}$ & $\begin{array}{l}\text { - O fator de crescimento de um tipo de bactéria é } 6 \text { (por } \\
\text { unidade de tempo }) \text {. No momento há 4 bactérias. } \\
\text { Calcular o momento em que haverá 100 bactérias. }\end{array}$ \\
\hline $\begin{array}{l}\text { 3. O contexto exerce no problema uma } \\
\text { função essencial e relevante. }\end{array}$ & $\begin{array}{l}\text { - Qual destes seria uma estimativa razoavelmente bóa } \\
\text { para a largura de uma sala de aula? () 4 pés () 10 pés } \\
\text { () 25 pés () 300 pés. }\end{array}$ \\
\hline
\end{tabular}

Quadro 2 - Classe de funcionalidade do contexto segundo De Lange (1995) Fonte: Elaborado pelas autoras com base em De Lange (1995).

Com base nos três níveis de funcionalidade de contexto apresentados por De Lange (1995), Meyer et al. (2001, p. 523) destacam cinco papéis do contexto no ensino e 
aprendizagem da que são muitas vezes interativos: motivar os alunos para explorar nova Matemática; oferecer a eles a oportunidade de aplicar a Matemática; servir como uma fonte de Matemática nova; sugerir uma fonte de estratégia de solução; fornecer uma âncora para a compreensão matemática.

No que diz respeito à importância da utilização de problemas de contexto, Dekker e Querelle (2002) apresentam algumas razões: (a) para introduzir um novo assunto ou conceito em Matemática - por meio de exemplos dentro de um contexto, o conteúdo matemático envolvido pode tornar-se mais claro; (b) para praticar um novo conceito ou procedimento resolvendo muitos problemas de contexto diferentes com mesmo conteúdo matemático, os estudantes podem aprender a utilizar e aplicar o conteúdo; (c) para mostrar o poder da Matemática - ao compreender que diferentes problemas de contexto podem envolver o mesmo conteúdo matemático; (d) para envolver os estudantes no problema - usando problemas da vida real, eles podem mostrar que são matematicamente alfabetizados e que sabem como a Matemática é utilizada para resolver problemas que surgem em situações da vida real.

Em uma tentativa de clarificar o conceito de problema, Borasi (1986) faz uma análise de vários exemplos a partir de quatro conceitos relacionados: (a) a formulação de um problema, isto é, a definição da tarefa a ser executada, (b) o contexto em que o problema está inserido, (c) o conjunto de soluções adequadas, (d) os métodos de abordagem que poderiam ser empregados na resolução do problema. De acordo com os quatro conceitos relacionados e suas características, a autora classifica os problemas em sete agrupamentos, que são apresentados parcialmente ${ }^{11}$ no quadro a seguir juntos de suas relações com o contexto (item b) e exemplos.

\begin{tabular}{|c|c|c|}
\hline Rótulo & Contexto & Exemplos \\
\hline Exercício & Inexistente & Encontre o resultado de $4 \times 2+6 \times 3$. \\
\hline $\begin{array}{c}\text { Problema de } \\
\text { palavra }\end{array}$ & $\begin{array}{l}\text { Totalmente } \\
\text { explícito no } \\
\text { texto }\end{array}$ & $\begin{array}{l}\text { Maria comprou um hambúrguer por } \$ 0.90 \text { e uma coca por } \\
\$ 0.30 \text { dólares. Se a taxa local sobre as vendas é de } 5 \% \text {, quanto ela } \\
\text { deverá receber se ela der ao caixeiro } \$ 2,00 \text { ? } \\
\text { (KANTOWSKI, } 1981 \text { apud BORASI, 1986). }\end{array}$ \\
\hline $\begin{array}{l}\text { Problema- } \\
\text { Enigma }\end{array}$ & $\begin{array}{l}\text { Totalmente } \\
\text { explícito no } \\
\text { texto }\end{array}$ & $\begin{array}{l}\text { Seis palitos de fósforos devem ser montados para formar quatro } \\
\text { triângulos equiláteros congruentes nos quais cada lado é igual ao } \\
\text { comprimento dos palitos. } \\
\text { (SCHEERER, } 1963 \text { apud BORASI, 1986). }\end{array}$ \\
\hline $\begin{array}{l}\text { Prova de uma } \\
\text { conjectura }\end{array}$ & $\begin{array}{l}\text { Apenas } \\
\text { parcialmente } \\
\text { no texto - }\end{array}$ & Prove que a fórmula: \\
\hline
\end{tabular}

\footnotetext{
${ }^{11}$ Apresentamos aqui apenas o item (b), e os itens (a), (c) e (d) na seção seguinte.
} 


\begin{tabular}{|c|c|c|}
\hline & $\begin{array}{l}\text { teorias } \\
\text { conhecidas são } \\
\text { assumidas }\end{array}$ & $\begin{array}{l}\qquad \begin{array}{l}a=2 m n \\
b=m^{2}-n^{2} \\
c=m^{2}+n^{2}\end{array} \\
\text { para qualquer par de números naturais } n \text { e } m \text { fornece todas as } \\
\text { soluções integrais da forma } \alpha^{2}+b^{2}=c^{2} \text {. }\end{array}$ \\
\hline $\begin{array}{l}\text { Problema da } \\
\text { vida real }\end{array}$ & $\begin{array}{l}\text { Apenas } \\
\text { parcialmente } \\
\text { no texto }\end{array}$ & $\begin{array}{l}\text { A esposa de Hans está morrendo. Um farmacêutico descobriu um } \\
\text { remédio que pode curar a sua doença, mas vende-o por um preço } \\
\text { que Hans não pode pagar. Hans deveria roubar o remédio? } \\
\text { (KOHLBERG, 1981, p. } 12 \text { apud BORASI, 1986). }\end{array}$ \\
\hline $\begin{array}{c}\text { Situação }{ }^{12} \\
\text { problemática }\end{array}$ & $\begin{array}{l}\text { Apenas } \\
\text { parcialmente } \\
\text { no texto - } \\
\text { problemático }\end{array}$ & $\begin{array}{l}\text { Você está no final de sua terceira década de vida, os seus filhos } \\
\text { estão bem na escola, seu marido está criando um nome para si } \\
\text { próprio na sua profissão e você está aborrecida. } \\
\text { (ADAMS, 1974, p. } 21 \text { apud BORASI, 1986). }\end{array}$ \\
\hline Situação & $\begin{array}{l}\text { Apenas } \\
\text { parcialmente } \\
\text { no texto - não } \\
\text { problemático }\end{array}$ & 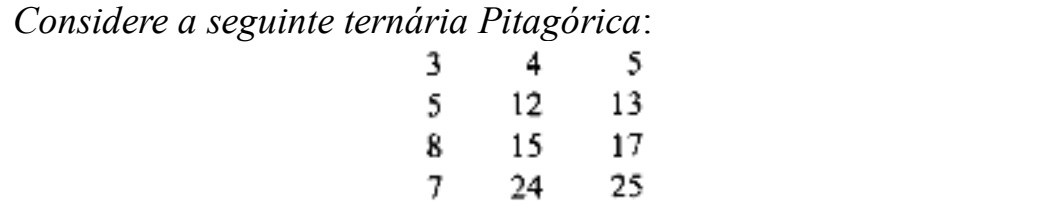 \\
\hline
\end{tabular}

Quadro 3 - Agrupamento do tipo de contexto conforme Borasi (1986)

Fonte: Elaborado pelas autoras com referência em Borasi (1986).

Segundo tal classificação, a existência de contexto parece estar relacionada ao "grau" em que uma situação é apresentada no enunciado, de uma forma mais geral, diz respeito à “situação na qual o problema está embutido" (BORASI, 1986, p. 129).

O projeto PISA ${ }^{13}$, segundo o documento INEE (2005), pretende dar possibilidade de que a intuição e a compreensão matemática dos estudantes sejam avaliadas em diferentes situações $^{14}$. Documentos do PISA (OECD, 2003; OECD, 2004a; OECD, 2006; OECD, 2010), em geral, classificam as situações em quatro ou cinco grupos. Para o PISA (OECD, 2006), a situação é a parte do mundo do estudante em que as tarefas se situam e está localizada a certa distância do estudante. A situação mais próxima é a da vida pessoal do estudante, em seguida vêm a da vida escolar, da vida profissional e de lazer, depois a da comunidade local e a sociedade. Situações científicas são as mais distantes ${ }^{15}$.

\footnotetext{
${ }^{12}$ Entendemos que Borasi (1986) chama de Situação uma tarefa que não apresenta explicitamente uma questão, pergunta. A tradução é literal e diferente do significado que atribuímos para situação ao longo do nosso texto.

${ }^{13}$ Maiores informações sobre o PISA podem ser encontradas no site: http://portal.inep.gov.br/pisa-programainternacional-de-avaliacao-de-alunos.

${ }_{15}^{14}$ Em alguns documentos também chamadas de contextos.

15 Em Ferreira (2013), é possível encontrar um quadro com algumas considerações sobre os cinco tipos de situações de tarefas: (1) uso pessoal ou privado; (2) pública; (3) ocupacional ou profissional; (4) educacional; (5) científica. O quadro foi construído com base nos documentos do PISA (OECD, 2003; OECD, 2004a; OCDE, 2005; OECD, 2006; OECD, 2010) e em De Lange (2003).
} 
O uso de situações nos contextos das tarefas de Matemática do PISA está relacionado à possibilidade de fornecer um contexto autêntico ${ }^{16}$ para o uso da Matemática.

\subsection{Caracterizações dos tipos de tarefas}

$\mathrm{Na}$ abordagem da Resolução de Problemas, os alunos deveriam ser responsáveis por decisões que, no passado, eram da responsabilidade de professores e livros didáticos. De acordo com McIntosh e Jarrett (2000), ao aluno cabe decidir que método ou procedimento utilizará, que estratégias tem possibilidade de desenvolver. Ele deve contar com suas experiências anteriores, esboçar uma linha de raciocínio, arquitetar uma resolução de modo autônomo ou com seus pares. Ao professor, cabe a responsabilidade de selecionar e lhe propor boas tarefas. Com isso, o docente deve criar condições para que os estudantes possam envolver-se, significativamente, com elas (MCINTOSH; JARRETT, 2000). Para além de fornecer respostas corretas, a Resolução de Problemas preocupa-se com os meios para a obtenção das respostas, com a elaboração de conhecimento por meio da tarefa proposta.

Nesse sentido, conhecer as oportunidades que as tarefas podem oferecer quanto aos possíveis métodos de solução, a pertinência das múltiplas respostas, os conceitos envolvidos, a familiaridade do estudante com a tarefa, o que lhe é solicitado em relação ao conteúdo ou às competências parecem se constituir em um recurso necessário que o professor precisa conhecer.

Consideramos que a escolha das tarefas, seja em uma situação de aula ou de avaliação, deve estar fortemente associada aos objetivos didáticos, os quais, por seu turno, determinarão que tarefas são potenciais para atingi-los. Quando o objetivo do professor for, por exemplo, avaliar o desempenho dos estudantes na mera reprodução de técnicas já conhecidas, uma boa escolha é a proposição de tarefas rotineiras, com as quais os alunos tenham intimidade e possam demonstrar sua competência, com pouca chance de terem como obstáculo a interpretação.

No quadro a seguir, são apresentadas algumas características para as tarefas do tipo rotineiras ou não-rotineiras.

\footnotetext{
${ }^{16}$ Autêntico no sentido de fazer sentido, de ser legítimo para o estudante, não necessariamente verdadeiro. Ainda que seja um contexto de conto de fadas, por exemplo, deve ser razoável com os fatos, fundamentado em algo, verossímil.
} 


\begin{tabular}{|c|c|}
\hline Rotineiras & Não-Rotineiras \\
\hline $\begin{array}{l}\text { Tarefas que são frequentes nas salas } \\
\text { de aula e nos livros didáticos } \\
\text { (BURIASCO, 1999). }\end{array}$ & $\begin{array}{l}\text { Tarefas que são muito pouco ou quase nunca frequentes nas } \\
\text { salas de aula e nos livros didáticos (BURIASCO, 1999). }\end{array}$ \\
\hline $\begin{array}{l}\text { Habitualmente, exercícios de } \\
\text { repetição e consolidação de } \\
\text { técnicas e de aplicação direta de } \\
\text { conhecimento (SANTOS, 2004). }\end{array}$ & $\begin{array}{l}\text { Entendem-se por experiências matemáticas de } \\
\text { aprendizagem situações que, aos olhos dos alunos, são } \\
\text { não-rotineiras e que apresentam certo nível de } \\
\text { complexidade. Entre elas, podemos ter a resolução de } \\
\text { problemas, tarefas de investigação, realização de projetos } \\
\text { e jogos (SANTOS, 2003). }\end{array}$ \\
\hline $\begin{array}{l}\text { Exercícios de rotina são } \\
\text { organizados para proporcionar } \\
\text { prática em uma determinada } \\
\text { técnica matemática que, } \\
\text { normalmente, acabou de ser } \\
\text { demonstrada para o aluno } \\
\text { (SCHOENFELD, 1992). }\end{array}$ & $\begin{array}{l}\text { Os problemas não-rotineiros incluem problemas de } \\
\text { processo (problemas para os quais não há nenhum } \\
\text { algoritmo padrão para extrair ou representar a informação } \\
\text { dada) e problemas com informação supérflua ou } \\
\text { insuficiente. A instrução foca em problemas passíveis de } \\
\text { estratégias particulares e inclui jogos (SCHOENFELD, } \\
\text { 1992). }\end{array}$ \\
\hline & $\begin{array}{l}\text { Problemas não rotineiros, na RME, são situações } \\
\text { genuínas de resolução de problema, para as quais o } \\
\text { estudante terá de trazer todo o conhecimento informal e } \\
\text { as estratégias que ele possui (GRAVEMEIJER, 1994). }\end{array}$ \\
\hline
\end{tabular}

Quadro 4 - Tarefas rotineiras e não-rotineiras.

Fonte: Elaborado pelas autoras.

De acordo com o Quadro 4, concluímos que as classificações limitam-se, quase sempre, à familiaridade do estudante com a tarefa proposta; quando conhecida, acaba sendo praticamente um "exercício", ao passo que, quando não "familiar", por alguma razão, quase sempre tem o caráter de problema. Entretanto, parece não haver uma distinção tão clara entre as duas ideias.

Butts (1997) apresenta uma classificação para cinco tipos típicos de problemas (para nós, tarefas) de matemática. São apresentados, no quadro a seguir, os tipos de tarefa segundo a classificação de Butts (1997), seguidos de suas definições.

\begin{tabular}{|l|l|}
\hline Tipo de tarefa & Definição \\
\hline $\begin{array}{l}\text { Exercício de } \\
\text { reconhecimento }\end{array}$ & $\begin{array}{l}\text { Usualmente, pede ao resolvedor para reconhecer ou recordar um fato } \\
\text { específico, uma definição ou enunciado de um teorema. }\end{array}$ \\
\hline $\begin{array}{l}\text { Exercícios } \\
\text { algorítmicos }\end{array}$ & $\begin{array}{l}\text { Exercícios que podem ser resolvidos com um procedimento passo a } \\
\text { passo, frequentemente, um algoritmo numérico já conhecido. }\end{array}$ \\
\hline $\begin{array}{l}\text { Problemas de paran para } \\
\text { aplicação }\end{array}$ & $\begin{array}{l}\text { São os que precisam da mudança da linguagem escrita com palavras param utilizar os } \\
\text { uma linguagem matemática adequada de modo que se possam } \\
\text { algoritmos apropriados. Os problemas tradicionais caem nesta categoria, } \\
\text { exigindo na sua resolução: (a) formulação do problema simbolicamente e } \\
\text { depois (b) manipulação dos símbolos mediante algoritmos diversos. }\end{array}$ \\
\hline $\begin{array}{l}\text { Problemas de } \\
\text { pesquisa aberta }\end{array}$ & $\begin{array}{l}\text { Problemas de pesquisa aberta são aqueles em cujo enunciado não } \\
\text { apresenta indícios de estratégia para resolvê-los. Usualmente, tais }\end{array}$ \\
\hline
\end{tabular}




\begin{tabular}{|l|l|}
\hline & $\begin{array}{l}\text { problemas expressam-se por: prove que, encontre todos, ou para quais, } \\
\text { mais outras muitas variações. }\end{array}$ \\
\hline $\begin{array}{l}\text { Situação- } \\
\text { Problema }\end{array}$ & $\begin{array}{l}\text { Neste subconjunto, não estão incluídos problemas propriamente ditos, } \\
\text { mas situações, nas quais uma das etapas decisivas é identificar o(s) } \\
\text { problema(s) inerente(s) à situação. }\end{array}$ \\
\hline
\end{tabular}

Quadro 5 - Classificação das tarefas segundo Butts (1997)

Fonte: Elaborado pelas autoras com referência em Butts (1997, p. 32-44).

De acordo com Butts (1997), uma alta porcentagem de exercícios e problemas propostos em livros didáticos recai nas três primeiras categorias, que quase sempre contêm em seu enunciado uma estratégia para resolvê-los ${ }^{17}$. Consequentemente, o obstáculo a se resolver é a tradução da tarefa para uma forma matemática apropriada e, a posteriori, a aplicação de algoritmos adequados.

Borasi (1986) também apresenta uma classificação para problemas (tarefas) de Matemática orientada por conceitos relacionados: exercício, problemas ou situação com base no contexto $^{18}$, tipo de formulação, soluções e métodos de abordagem. Essa classificação é apresentada no quadro a seguir.

\begin{tabular}{|l|l|l|l|}
\hline \multicolumn{1}{|c|}{ Tipo da tarefa } & \multicolumn{1}{|c|}{ Formulação } & \multicolumn{1}{|c|}{ Soluções } & \multicolumn{1}{c|}{ Métodos de abordagem } \\
\hline Exercício & Única e explícita & $\begin{array}{l}\text { Sobretudo única e } \\
\text { exata }\end{array}$ & Combinação de algoritmos conhecidos \\
\hline $\begin{array}{l}\text { Problema de } \\
\text { palavra }\end{array}$ & Única e explícita & $\begin{array}{l}\text { Sobretudo única e } \\
\text { exata }\end{array}$ & Combinação de algoritmos conhecidos \\
\hline $\begin{array}{l}\text { Problema- } \\
\text { Enigma }\end{array}$ & Única e explícita & $\begin{array}{l}\text { Sobretudo única e } \\
\text { exata }\end{array}$ & $\begin{array}{l}\text { Elaboração de um novo algoritmo - ato } \\
\text { de reformulações-reflexivas }\end{array}$ \\
\hline $\begin{array}{l}\text { Prova de uma } \\
\text { conjectura }\end{array}$ & Única e explícita & $\begin{array}{l}\text { Geralmente, mas não } \\
\text { necessariamente } \\
\text { única }\end{array}$ & $\begin{array}{l}\text { Reformulação do contexto - } \\
\text { reformulações - elaboração de novos } \\
\text { algoritmos }\end{array}$ \\
\hline $\begin{array}{l}\text { Problema da Vida } \\
\text { real }\end{array}$ & $\begin{array}{l}\text { Parcialmente dada - } \\
\text { muitas alternativas } \\
\text { possíveis. }\end{array}$ & $\begin{array}{l}\text { Muitas possíveis - } \\
\text { apenas soluções } \\
\text { aproximadas }\end{array}$ & $\begin{array}{l}\text { Reformulação do contexto - } \\
\text { reformulações - criação de um modelo }\end{array}$ \\
\hline $\begin{array}{l}\text { Situação } \\
\text { problemática }\end{array}$ & $\begin{array}{l}\text { Muitas implicitamente } \\
\text { sugeridas - uma } \\
\text { explícita pode ser dada }\end{array}$ & $\begin{array}{l}\text { Muitas possíveis } \\
\text { Reformulação do contexto - } \\
\text { reformulações - problematização }\end{array}$ \\
\hline Situação & $\begin{array}{l}\text { Inexistente - nem } \\
\text { mesmo implicitamente }\end{array}$ & $\begin{array}{l}\text { A criação de um } \\
\text { problema }\end{array}$ & Problematização \\
\hline
\end{tabular}

Quadro 6 - Classificação de Problemas - Borasi (1986)

Fonte: Adaptado de Borasi (1986, p. 134, tradução nossa).

Uma análise da natureza dos problemas (tarefas) é necessária para avaliar e explorar seu alcance e a significância na área da educação. Dada a natureza complexa e discutível desse assunto, o objetivo é iniciar uma investigação para estimular o debate (BORASI, 1986).

\footnotetext{
${ }^{17}$ Vale a pena observar que, apesar de a publicação de Butts (1997) ter mais de 15 anos, a afirmação do autor de que a maioria dos problemas contidos nos livros didáticos, de qualquer nível, pertencia aos três primeiros agrupamentos, aparentemente, não mudou.

${ }_{18}^{18}$ Apresentado na seção anterior.

${ }^{19} \mathrm{O}$ que diferencia aqui problema de palavra e exercício é que esse não apresenta contexto algum em seu enunciado.
} 
No que diz respeito à formulação da tarefa e às possibilidades de solução, os documentos do PISA (OECD, 2004b; OECD, 2004c; OECD, 2005) apresentam a seguinte classificação relativa aos tipos de itens ${ }^{20}$ :

1) Múltipla Escolha: com alternativas - envolve a escolha de uma alternativa das apresentadas. A resolução trata-se apenas de indicar uma resposta.

2) Múltipla Escolha Complexa: com alternativas - envolve escolher as alternativas de vários itens propostos. Para cada item, os alunos devem escolher uma resposta para cada opção apresentada.

3) Resposta de Construção Fechada: sem alternativas - permite a construção livre de respostas com limite fechado de respostas aceitáveis. Os tipos de respostas são similares às dos tipos de respostas em múltipla escolha.

4) Resposta Curta: sem alternativas - permite a construção livre de respostas com maior amplitude de possibilidades de respostas possíveis. Respostas breves.

5) Resposta de Construção Aberta: sem alternativas - exigem respostas mais longas, sofisticadas, como pedido de explicação, argumentação, justificativa, opinião, pontos de vista, relacionamento de ideias, conexões, reflexão.

Kirkley $(2003)^{21}$ apresenta três agrupamentos de problemas no que concerne às suas características de resolução e possibilidades de resposta. A autora chama de Problemas bem estruturados aqueles que sempre usam a mesma solução passo a passo, nos quais a estratégia de solução é usualmente previsível. Problemas moderadamente estruturados exigem uma variedade de estratégias e adaptações para se adequar a contextos particulares. Nesse tipo de problema, muitas vezes, é aceitável mais do que uma estratégia de solução. Problemas de estrutura imperfeita possuem objetivos vagos e imprecisos e as estratégias de solução são menos restritas e as soluções não são únicas e bem definidas anteriormente (KIRKLEY, 2003).

\subsection{A respeito das características das tarefas a partir da análise de seus contextos}

\footnotetext{
${ }^{20}$ Os tipos de itens apresentados pelo PISA dizem respeito, especificadamente, às tarefas de avaliação. Entretanto, podem servir para estender a discussão no que diz respeito às tarefas de sala de aula de modo geral.

${ }^{21}$ Com base em Newell e Simon (1972) - NEWELL, A.; SIMON, H. Human Problem Solving. Englewood Cliffs, NJ: Prentice Hall, 1972.
} 
Considerando que não deveria haver diferenças entre tarefas de sala de aula e de avaliação, conforme discutido anteriormente, reconhecemos que há, no entanto, diferenças no que diz respeito aos seus objetivos, contexto de aplicação, duração das tarefas, autonomia. Com isso, considera-se que algumas características de bons problemas (tarefas) de avaliação, na perspectiva da RME, indicadas por Van den Heuvel-Panhuizen (1996, 2005), também devem ser consideradas para as tarefas de sala de aula. Para a autora, bons problemas de avaliação devem ser informativos, significativos, transparentes, elásticos/flexíveis, acessíveis.

O caráter informativo é relativo à possibilidade de o problema fornecer o máximo de informações a respeito do conhecimento dos estudantes, insights, competências, incluindo suas estratégias, procedimentos, formas de raciocínio. Em outras palavras, problemas informativos devem fornecer uma imagem o mais completa possível do aprendizado dos estudantes.

Para que eles possam mostrar suas formas genuínas de lidar com os problemas, as situações problemáticas devem ser significativas. Isso significa que os problemas devem ser convidativos para o aluno, que valha a pena resolver, desafiadores e que sua solução seja útil para o fornecimento de uma ou várias respostas. Uma forma de avaliar se um problema é significativo reside nas respostas das perguntas: o estudante pode se sentir o dono do problema, aquele que domina a situação? Ele pode, a partir da situação, se colocar a pensar a respeito de questões próprias? Por outro lado, para que os problemas sejam significativos do ponto de vista da avaliação, eles devem refletir objetivos importantes, se alguma coisa não apresenta motivo para ser aprendida, não é útil para a avaliação (VAN DEN HEUVELPANHUIZEN, 1996).

Para que o caráter significativo possa se revelar, os problemas devem ser antes acessíveis aos estudantes. Isso significa que os enunciados devem ser tão claros quanto possível, de forma que os estudantes possam, pelo menos, refletir sobre o assunto nele envolvido. Tal aspecto não significa que esses problemas devem sugerir estratégias ou a indicação de solução, mas permitir que o estudante possa, a seu nível, revelar a forma pela qual abordaria o problema.

Ao encontro da acessibilidade dos problemas, o caráter de transparência deve permitir ao estudante mostrar o nível em que se encontra. Nesse sentido, um problema não pode ser tão fechado a ponto de ser resolvido por uma única maneira, de modo a impedir que o estudante demonstre suas habilidades, ainda que seja por meio de seus métodos informais.

Para que a avaliação seja tão transparente quanto possível, o caráter de flexibilidade e o de elasticidade sugerem que os problemas possam ser resolvidos por diferentes estratégias, 
em diferentes níveis de aprendizagem. Os alunos devem ter a oportunidade de dar suas respostas por suas próprias palavras (VAN DEN HEUVEL-PANHUIZEN, 1996).

No âmbito dessas características de bons problemas de avaliação, o papel dos contextos é essencial, pois "comparado com problemas numéricos simples, problemas de contexto oferecem aos estudantes mais oportunidades de demonstrar suas habilidades" (VAN DEN HEUVEL-PANHUIZEN, 1996, p. 94). No entanto, não é apenas o fato de apresentar alguma situação ou contexto no texto do problema que o faz tornar-se mais flexível. Para Van den Heuvel-Panhuizen (2005), os problemas de palavras são frequentemente confundidos com problemas de contexto. Para a autora, os problemas de palavras refletem um contexto não muito essencial. Repetidas vezes, nesse tipo de problema, o contexto é utilizado apenas para camuflar, embalar um contexto matemático. Nesse sentido, é possível despir facilmente o problema matemático envolvido na situação realizando simples traduções para a linguagem simbólica.

Tão inflexíveis quanto os problemas de palavras podem ser, os problemas nus ${ }^{22}$, despidos são aqueles que não envolvem contexto algum. Esses problemas dão pouca liberdade na forma de abordagem. Quase sempre, formulam-se a partir de expressões como resolva as seguintes equações, qual o par ordenado é solução para o sistema. Para De Lange (1999), esse tipo de problema deve ser evitado.

De Lange (1999), considerando as características para bons problemas de avaliação, na perspectiva da RME, apresenta uma "pirâmide de avaliação" (ver Figura 1) para fornecer uma imagem visual dos problemas que são necessários para representar o processo no qual um estudante se encontra.

No Nivel $I$, as tarefas demandam resumidamente competências relacionadas à reprodução como: reconhecer fatos, aplicar algoritmos conhecidos, desenvolver habilidades técnicas, reconhecer equivalências, recordar objetos matemáticos e propriedades, realizar procedimentos de rotina. As tarefas nesse nível, geralmente, são do tipo de Múltipla Escolha, Múltipla Escolha Complexa, de Resposta de Construção Fechada (DE LANGE, 1999). Geralmente, as questões são isoladas sem muita ligação com situações reais ou imaginárias.

As tarefas de Nível II envolvem competências de conexão. Demandam que os estudantes lidem com diferentes formas de representação de acordo com a situação, integrem informações, sejam capazes de distinguir e relacionar diferentes declarações, de decodificar e interpretar linguagem simbólica ou formal, bem como relacioná-las com a linguagem natural,

\footnotetext{
${ }^{22}$ Do inglês "bare problem".
} 
formular e resolver problemas e lidar com situações (DE LANGE, 1999). Nesse nível, as tarefas demandam alguma forma de matematização e, são geralmente, do tipo de Resposta Curta ou Resposta de Construção Fechada. Segundo De Lange (1999), frequentemente, as tarefas desse tipo são colocadas dentro de um contexto de envolver os alunos na tomada de decisão matemática.

As tarefas de Nível III (reflexão) envolvem a matematização de situações que, segundo De Lange (1999), reside no conhecimento e na extração da Matemática envolvida, bem como sua utilização para a resolução do problema. Envolvem análise, interpretação, desenvolvimento de modelos e estratégias, proposição de questões, apresentação de argumentação, provas, generalizações, incluem ainda reflexão a respeito de todo o processo (DE LANGE, 1999). Tarefas do tipo de Resposta de Construção Aberta são mais características desse nível.

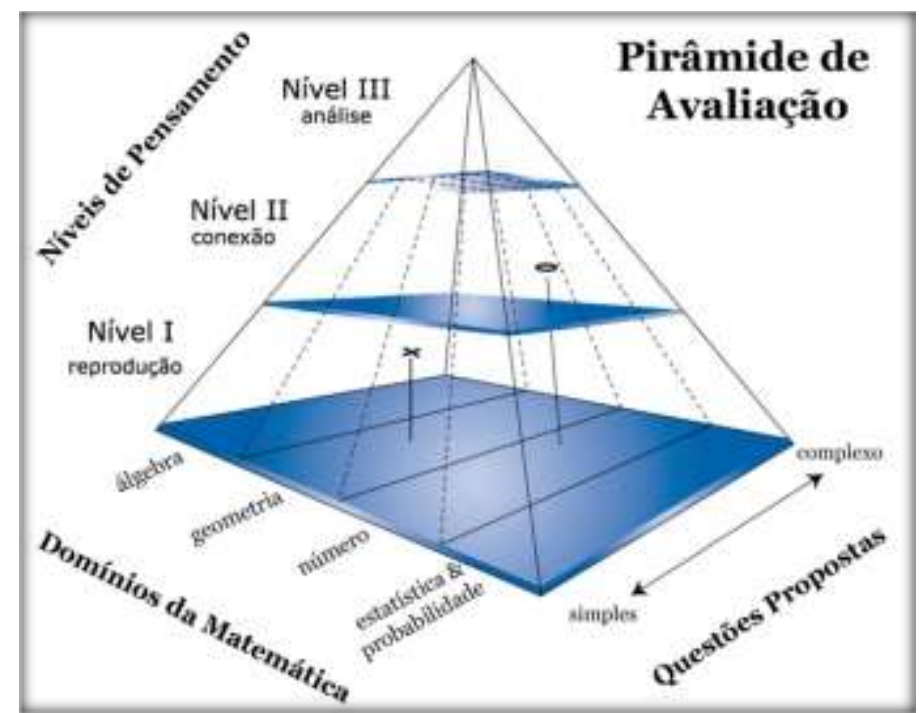

Figura 1 - Pirâmide de Avaliação proposta por De Lange (1999)

Fonte: Traduzida e adaptada de Shafer e Foster (1997, p. 3).

De Lange (1999) salienta que a definição dos três níveis é uma ação um tanto arbitrária dado que não há uma distinção tão clara entre eles. Pode ocorrer de alguma tarefa, em algum nível, incorporar competências associadas a outro nível. A pirâmide fornece uma imagem visual justa da quantidade de tarefas necessárias para representar o desempenho dos estudantes (DE LANGE, 1999). Assim, concentra-se em uma quantidade maior no Nível I, seguida pelos dois outros níveis, considerando que o tempo e qualidade do lidar com tarefas do Nível III são, relativamente, maiores do que nas tarefas de nível II e I. Nos diferentes 
níveis, as tarefas ainda podem variar de simples a complexas $^{23}$ ou ainda do tratamento informal para oformal.

\section{Considerações finais}

Propor aos estudantes tarefas matemáticas que apresentem contextos diversos é uma alternativa para que possam ampliar seus conhecimentos, pois, mais do que aprender a operar dados, o ensino da Matemática deveria propiciar que os alunos pudessem resolver tarefas com mais referência em sua realidade do que aquelas apenas do tipo efetue, some, divida, calcule a seguinte regra de três, apresentadas rotineiramente nas escolas. Até porque a aprendizagem escolar pode se constituir como uma base para que nossos alunos continuem aprendendo, dentro e fora da escola, para que tenham uma participação efetiva na sociedade.

No que diz respeito à classificação das tarefas com base no agrupamento de Díaz e Poblete (2005), Ferreira (2013), que estudou tarefas matemáticas em um livro didático tradicional, observa que os contextos das tarefas explorados no seu estudo limitam-se apenas a dois grupos: ser puramente matemáticos ou revestidos de alguma situação artificial com referência na realidade. Os problemas de contextos reais (que podem ser produzidos efetivamente na realidade) e os fantasiosos não são explorados. Por esse motivo, dentre outros, acreditamos que estabelecer relações entre os conteúdos matemáticos aprendidos na escola e suas utilidades em situações reais, fora dela, parece tão difícil aos estudantes. A referência obtida se resume, quase sempre, em aprender Matemática de forma técnica, mecânica ou, no máximo, inserida em situações artificiais.

Há de se levar em consideração que não é o fato de uma tarefa apresentar alguma situação real, ou com referência na realidade, ou ainda fictícia que faz com que ela venha a se constituir em um Problema de Contexto. Com isso, autores como De Lange (1999) e Van den Heuvel-Panhuizen $(1996,2005)$ justificam a relevância do contexto para a resolução do problema. Se a questão pode ser facilmente despida e resolvida sem o uso da situação, muito provavelmente estamos lidando com um Problema de Palavras, cuja situação pode ser substituída por outras análogas.

\footnotetext{
${ }^{23}$ No original, o autor utiliza os termos "fácil" e "difícil", os quais, consideramos nós, serem relativos à forma como o sujeito enfrenta as tarefas. Acreditamos ser mais coerente a utilização dos termos "simples" e "complexos" pelo fato de essas características serem associadas às tarefas e não aos sujeitos.
} 
Um bom Problema de Contexto é o que conta com a característica de a situação ser autêntica para a resolução do estudante. Desse ponto de vista, o assunto matemático e a situação são dificilmente separáveis. O caráter da autenticidade vai ao encontro do que a Educação Matemática Realística propõe: a Matemática no ambiente de ensino e aprendizagem deve emergir da exploração de fenômenos que são experiencialmente reais, mais especificamente, realísticos. Real não no sentido de ser factual, verdadeiro, mas real no sentido de ser imaginável, concebido, concreto (por ter passado pelo processo de abstração ${ }^{24}$ ), verossímil. Por esse motivo, acreditamos que a tradução para realístico é mais apropriada.

A autenticidade de uma tarefa está fortemente ligada à relação que o sujeito estabelecerá com ela. Portanto, é possível acontecer de um bom Problema de Contexto (aos olhos do educador) não representar um problema (que valha a pena ser resolvido) para o estudante.

Contudo, considerar que, mesmo que uma tarefa envolva situação de fato real, não é condição suficiente para ser um Problema de Contexto se o estudante não reconhecer na situação a relevância para a resolução. Nesse caso, pode-se dizer que o problema é de contexto camuflado, serve apenas para promover a Matemática desejada.

Com relação à relevância do contexto para o problema, De Lange $(1987,1995,1999)$ estabelece alguns critérios, que se relacionam tanto com a importância que o contexto desempenha na resolução quanto com a oportunidade de matematização. Com isso, a possibilidade de matematizar parece estar fortemente associada ao papel que o contexto desempenha no sentido de que quanto mais realístico o contexto proposto for, mais o estudante tem a oportunidade de torná-lo familiar e, por conseguinte, produzir a sua Matemática.

Consideramos que o enunciado de uma tarefa, por si só, não garante a possibilidade ou não de matematização. $\mathrm{O}$ enunciado da tarefa é apenas um mote, e para que sua execução oportunize a matematização deve-se considerar também o contexto prático no qual a tarefa é empregada, o tratamento e a situação na qual o professor trabalha com ela. Tal aspecto pode ser motivo de análises futuras.

\section{Referências}

BARDIN, L. Análise de conteúdo. 3 ed. Lisboa: Edições 70 Ltda., 2004.

\footnotetext{
${ }^{24}$ KOSIK, K. Dialética do Concreto, $2^{\text {a }}$ ed., São Paulo: Paz e Terra, 1976.
} 
BORASI, R. On the nature of problems. Educational Studies in Mathematics, v. 17, n. 2, p. 125$141,1986$.

BURIASCO, R. L. C. de. Avaliação em Matemática: um estudo das respostas de alunos e professores. 1999. 238 f. Tese (Doutorado em Educação) - Universidade Estadual Paulista, Marília, 1999.

BURIASCO, R. L. C.; FERREIRA, P. E. A.; CIANI, A. B. Avaliação como prática de investigação (alguns apontamentos). Bolema, Rio Claro, v. 22, n. 33, p. 69-96, 2009.

BUTTS, T. Formulando problemas adequadamente. In: KRULIK, S.; REYS, R. E. A Resolução de Problemas na Matemática Escolar. São Paulo: Atual, 1997. p. 32-48.

CLEMENTS, M. Analyzing children's errors on written mathematical tasks. Educational Studies in Mathematics, v. 11, n. 1, p. 1-21, 1980.

DE LANGE, J. Mathematics, Insight and Meaning. Utrecht: OW \&OC, 1987.

DE LANGE, J. Assessment: no change without problems. In: ROMBERG, T.A. (Ed.). Reform in School Mathematics. Albany, NY: SUNY Press, 1995.

DE LANGE, J. Framework for classroom assessment in mathematics. Madison: WCER, 1999.

DEKKER, T.; QUERELLE, N. Great assessment problems. Utrecht: Freudenthal Instituut, 2002. Disponível em: $<$ http://www.fisme.science.uu.nl/catch/products/GAP_book/intro.html $>$. Acesso em: 24 jun. 2014.

DÍAZ, V.; POBLETE, A. Competencias en Matemáticas y Tipos de problemas. In: CIBEM Proccedings V Congreso Iberoamericano de Educación Matemática, n. 5., 2005, Portugal. Anais... Portugal: Publicaciones con Comité Editorial, 2005.

FERREIRA, P. E. A. Análise da produção escrita de professores da Educação Básica em questões não-rotineiras de matemática. 2009. 166 f. Dissertação (Programa de Pós-Graduação em Ensino de Ciências e Educação Matemática) - Universidade Estadual de Londrina, Londrina, 2009.

FERREIRA, P. E. A. Enunciados de Tarefas de Matemática: um estudo sob a perspectiva da Educação Matemática Realística. 2013. 121 f. Tese (Programa de Pós-Graduação em Ensino de Ciências e Educação Matemática) - Universidade Estadual de Londrina, Londrina, 2013.

FREUDENTHAL, H. Didactical phenomenology of mathematical structures. Dordrecht: Reidel Publishing Company, 1983.

GRAVEMEIJER, K. P. E. Developing realistic mathematics education. Utrecht: Utrecht University, 1994.

GRAVEMEIJER, K. P. E.; DOORMAN, M. Context problems in realistic mathematics education: a calculus course as an example. Educational Studies in Mathematics, v. 39, n. 1, p. 111-129, Jan. 1999.

INEE. PISA para docentes: la evaluación como oportunidad de aprendizaje. INEE - Instituto Nacional Para La Evaluación De La Educación, México, 2005.

KIRKLEY, J. Principles for teaching problem solving. USA: PLATO Learning Inc, 2003.

MCINTOSH, R.; JARRETT, D. Teaching mathematical problem solving: implementing the vision. 
Portland, Oregon: Mathematics and Science Education Center, North West Regional Laboratory, 2000. Disponível em: <http://www.cimm.ucr.ac.cr/ciaem/articulos/universitario/conocimiento/Teaching\%20 Mathematical\%20Problem\%20Solving:\%20Implementing\%20the\%20Vision*McIntosh,\%20Robert\% 20.*McIntosh.pdf $>$. Acesso em: 19 jun. 2014.

MEYER, M.; DEKKER, T.; QUERELLE, N. Context in mathematics curricula. Mathematics teaching in the middle school, v. 9, p. 522-527, 2001.

OECD. The PISA 2003 - Assessment Framework: Mathematics, Reading, Science and Problem Solving Knowledge and Skills. Paris, 2003. Disponível em: $<$ http://www.oecd.org/dataoecd/46/1 4/33694881.pdf >. Acesso em: 24 jun. 2014.

OECD. Estrutura de avaliação PISA 2003: conhecimentos e habilidades em matemática, leitura, ciências e resolução de problemas. Tradução B \& C Revisão de textos. São Paulo: Moderna, 2004a.

OECD. PISA 2003: conceitos fundamentais em jogo na avaliação de resolução de problemas. Lisboa: Ministério da Educação. Gabinete de Avaliação Educacional (GAVE), 2004b.

OECD. Resultados do Estudo Internacional PISA 2003. Lisboa: Ministério da Educação. Gabinete de Avaliação Educacional (GAVE), 2004c.

OECD. Aprendendo para o mundo de amanhã: primeiros resultados do PISA 2003. São Paulo: Moderna, 2005.

OECD. PISA Assessing Scientific, Reading and Mathematical Literacy: A Framework for PISA 2006. Paris: OECD Publications, 2006.

OECD. PISA 2012 Mathematics Framework. Paris: OECD Publications, 2010. Disponível em: $<$ www.oecd.org/dataoecd/8/38/46961598.pdf > . Acesso em: 09 jul. 2012.

SANTOS, L. Avaliar competências: uma tarefa impossível? Educação e Matemática, v. 74, p. 16-21, set/out. 2003.

SANTOS, L. A avaliação das aprendizagens em Matemática: orientações e desafios, 2004.

Disponível em: $<$ http://www.educ.fc.ul.pt/docentes/msantos/TextolivroPaulo.pdf $>$. Acesso em: 7 jun. 2014.

SCHOENFELD, A. H. Learning to Think Mathematically: Problem Solving, Metacognition, and Sense Making in Mathematics. In: GROUWS, D. A. (Ed.). Handbook of Research on Mathematics Teaching and Learning. New York: MacMillan, 1992. p. 334-370.

SHAFER, M. C.; FOSTER, S. The changing face of assessment. Principled Practice in Mathematics and Science Education, v. 1, n. 2, p. 1-8, 1997. Disponível em:

$<$ http://ncisla.wceruw.org/publications/newsletters/fall97.pdf $>$. Acesso em: 24 jun. 2014.

SHANNON, A. Task Context and Assessment. In: SCHOENFELD, A. H. (Ed.). Assessing Mathematical Proficiency. Cambridge: Cambridge University Press, 2007. Disponível em: $<$ http://library.msri.org/books/Book53/files/13shannon.pdf >. Acesso em: 24 jun. 2014.

STREEFLAND, L. Fractions in Realistic Mathematics Education. Dordrecht: Kluwer, 1991.

TREFFERS, Three Dimensions: a model of goal and theory description in mathematics instruction The Wiskobas Project. Dordrecht: Reidel Publishing Company, 1987.

TREFFERS, A.; GOFFREE, F. Rational analysis of realistic mathematics education. In: 
STREEFLAND, L. (Ed.). Proceedings of the 9th International Conference for the Psychology of Mathematics Education. Utrecht, The Netherlands: OW\&OC. 1985. p. 97-123.

VAN DEN HEUVEL-PANHUIZEN, M. V. D. Assessment and Realistic Mathematics Education. Utrecht: CD-B Press/Freudenthal Institute, Utrecht University. 1996.

VAN DEN HEUVEL-PANHUIZEN, M. V. D. Mathematics education in the Netherlands: A guided tour. Freudenthal Institute Cd-rom for ICME9. Utrecht: Utrecht University, 2000. CD-ROM.

VAN DEN HEUVEL-PANHUIZEN, M. V. D. Realistic Mathematics Education as work in progress. In: LIN, F. L. (Ed.). Common Sense in Mathematics Education. Proceedings of 2001 The Netherlands and Taiwan Conference on Mathematics. Taipei, Taiwan, p. 1-43, Nov. 2001. Disponível em: <http://www.fi.uu.nl/publicaties/literatuur/4966.pdf>. Acesso em: 24 jun. 2014.

VAN DEN HEUVEL-PANHUIZEN, M. V. D. The role of contexts in assessment problems in mathematics. For the Learning Mathematics, Alberta-Canadá, v. 25, n. 2, p. 2-9, 2005. Disponível em: <http://www.fi.uu.nl/ marjah/documents/01-Heuvel.pdf>. Acesso em: 12 ago. 2008.

VAN DEN HEUVEL-PANHUIZEN, M. V. D. Reform under attack - Forty Years of Working on Better Mathematics Education thrown on the Scrapheap? No Way! In: SPARROW, L.; KISSANE, B.; HURST, C. (Eds.). Proceedings of the 33th annual conference of the Mathematics Education Research Group of Australasia. Fremantle: MERGA, 2010.

Submetido em Junho de 2014. Aprovado em Dezembro de 2014. 